\title{
PREŠERENS EIGENE ÜBERSETZUNG DES SPÄTEREN MOTTOS PROSTO SRCE (DAS FREIE HERZ). MUTTERSPRACHE UND ZWEITSPRACHE IN EINEM DICHTERISCHEN KONTEXT
}

\section{Einige sprachwissenschaftliche Ausgangspunkte}

In der Sprachwissenschaft bekam der Strukturalismus eine bedeutende Stellung. Jedoch muß man den deskriptiven Strukturalismus als Beschreibung grammatischer Kennzeichen einzelner Äußerungen gegenüber dem Strukturalismus, der Sätze und Texte als einen Korpus einer natürlichen Sprache sieht, unterscheiden. Die linguistischen Strukturen und die ihnen zugrunde liegenden Gesetzmäßigkeiten, wie das die generative Grammatik zu erforschen sucht, sind vor allem eine Theorie, die eine menschliche Fähigkeit in einer einheitlichen Weise rational schematisch auffaßt und zergliedert.

Die kommunikativen Funktionen der Sprache berücksichtigen die psychologischen, soziologischen und kulturellen Elemente und erforschen strukturelle sprachliche Kennzeichen, die für gewisse Situationen oder aus gewissen Situationen gebildet werden.

Die grobe Einteilung der Sprache in "Subsprachen der praktischen, der emotionalen, der rhetorischen und der poetischen Sprache ${ }^{11}$ muß auch die jeweiligen stilistischen Mittel ergründen. Das ist aber nicht leicht, da man aus der sogenannten praktischen Sprache nicht die emotionalen und die rhetorischen Elemente ausschließen kann. Aber auch die poetischen Sprachmittel können nur auf den Strukturelementen der sog. "praktischen Sprache" gebildet werden. Was "poetisch" ist (ästhetisch ist schon ein zu breiter Begriff), glaubt man literaturtheoretisch zwar immer wieder mit (klassischen) poetologischen Maßstäben feststellen zu können. Wenn es gesagt wird, daß man bei der Untersuchung eines dichterischen Textes das Augenmerk auf "ein spezielles System von Zeichen mit interner ästhetischer Funktion"2 richten muß, ist das nur dann möglich,

1 Horst Albert Glaser: Literatur-Dichtung-Poesie. In: Grundzüge der Literatur- und Sprachwissenschaft. Band 1: Literaturwissenschaft. Herausgegeben von Heinz Ludwig, Arnold und Volker Sinemus. München. Deutscher Taschenbuch Verlag 1973, S. 37.

2 Ebenda, S. 87. 
wenn man ein Zeichensystem, das für jede Sprachverwendung da ist, überhaupt kennt. Weiters muß man die Sprachverwendungen kennen, daß man "das poetische" bestimmen kann. So gelangt man immer wieder zum sich wandelnden Begriff "poetisch".

Gleichzeitig gelangt man mit solchen Feststellungen zur Texttheorie, wo heutzutage ebenso Arten oder Sorten herausgebildet worden sind, die aber auch bei weitem nicht so einheitlich vorkommen, wie das einige Texttheorien zu behaupten suchen.

Bei der Textbildung muß es sich immer um die Bildung von Einheiten einer hierarchischen Stufe in der Dimension des sprachlichen Nacheinanders handeln, wo aber auch die Möglichkeit eines Statteinanders besteht, ohne daß dadurch die Etablierung der Textstufe zerstört wird.

Die Menschen äußern sich nicht in einzelnen Wörtern, auch nicht in Sätzen, sondern in Kon(Texten). Auch die sog. Einwortsätze, die einzelnen Sätze als Frage oder Antwort, Sprüche und Sentenzen mit einem einfachen oder komplexen Satz sind (kon)textgebunden.

So muß man in der Textlinguistik wirklich über zwei verschiedene Kategorien von Motiven sprechen: von den linguistik-internen und von den linguistik-externen. ${ }^{3}$

Die Sichten auf texttypologische Fundamentaloppositionen versucht man durch verschiedene Charakterisierungen der Texte zu veranschaulichen. Wir werden hier nur einige nennen: monologische-dialogische, wissenschaftliche-nichtwissenschaftliche, repräsentationale (mit realen, d.h., objektiv oder subjektiv feststellbaren Korrelaten in der Welt) - nichtrepräsentationale (mit keinen solchen Korrelaten); die letzteren werden wieder in fiktive (pseudoreale) und imaginative (mit nicht einmal pseudorealen Korrelaten) eingeteilt. ${ }^{4}$

Natürlich werden ganz einheitliche Strategien selten durchgehalten, aber es gibt doch Texte, an denen man eine Typologie durch Erforschungen von gewissen Gesetzmäßigkeiten feststellen kann. So kann man auch zugeben, daß eine der wichtigsten Bedeutungen der Textlinguistik darin liegt, daß sie die enge, satzbezogene Perspektive der Linguistik überwunden und damit eine Basis geschaffen hat zur Einbeziehung pragmatischer Aspekte und zur interdisziplinären Zusammenarbeit, besonders mit der Literaturwissenschaft. ${ }^{5}$

Es handelt sich immer um kommunikations- und situationsbedingte sprachliche Äußerungen mit gewissen psychologischen, soziologischen und im weitesten Sinne ästhetischen Gesetzen.

Bei der Untersuchung sind die literarischen Texte zunächst immer "sprachliche Objekte, die auf dem linguistischen Text-Rang zu beschreiben sind, und zwar nach

3 Roland Harweg: Textlinguistik. In: Perspektiven der Linguistik II. Herausgegeben von Walter A. Koch. Stuttgart. Alfred Kröner Verlag 1974, S. 89.

4 Ebenda, S. $108 \mathrm{f}$.

5 Hadumod Bußman: Lexikon der Sprachwissenschaft. Stuttgart. Alfred Kröner Verlag 1990, S. 779. 
ihren strukturellen und stilistischen Eigenschaften; zum anderen literarische und damit historische Objekte, die entsprechend den in der Systematik der Literaturwissenschaft definierten Beziehungen zu beschreiben sind." 6

Der literarische Text ist eine einheitliche Sprachhandlung, besonders muß dies in einem kurzen Text, wie das ein Motto ist, berücksichtigt werden. Es gibt da logisch-inhaltliche Beziehungen zwischen den Propositionen, ebenso referentielle Beziehungen zwischen den Argumenten. ${ }^{7}$ Die genetische innere sprachliche Struktur, die aber mittels der objektiven Gegebenheit der Struktur einer Sprache überhaupt nachweisbar ist, muß gefunden und zergliedert werden, daß man die Subjektivität des Dichters, der zur Objektivität mehr oder weniger eigenartig (einmalig) Stellung nimmt, analytisch und synthetisch gelangt. Die Eigenart der sprachlich ausgedrückten Beziehungen zwischen Inhalt (Gehalt) und Referenzen ergibt auch die poetische Erscheinung und den poetischen Wert.

Für jede sprachliche Kommunikation (für die Rezeption eines poetischen Textes noch mehr) gilt es, daß der Kommunikationspartner das System von sprachlichen Regeln (das Sprachsystem) der Sprache, in der ein Dichter schreibt, beherrscht. Das gilt desto mehr, wenn es sich um sprachliche Experimente handelt. Aber mit den letzteren werden wir uns hier nicht befassen.

Die Sprache ist immer intern- und extern-gebunden. Auch die Sprache eines Dichters kann man nur gut verstehen (oder besser verstehen), wenn man auch gewisse Faktoren von der Thematik, vom gesellschaftlichen Status des Dichters, seiner besprochenen oder besungenen Personen (der auftretenden und der nicht-auftretenden) kennt. Bedeutend sind auch gewisse soziale und psychologische Lagen des Dichters und seiner Personen (wenn von ihnen gesprochen wird), ihre kommunikativen Rollen und Schicksale, ihre Einstellungen usw. Die Kenntnisse können in alle Details übergehen oder nur bei den wichtigsten Elementen bleiben. Etwas muß der Rezipient wissen, dazu verhelfen ihm die zusätzlichen, außerliterarischen Informationen.

Weltbezug und Moralkodexe müssen ebenso verstanden werden.

Wenn man eine Sprache, die immer bestimmte soziokulturelle Elemente und die oben erwähnten Faktoren ausdrückt, weiter die speziellen Faktoren der Geschichte, von der die allgemeine und soziale Kultur des Landes geprägt ist, nicht oder schlecht kennt, kann es zu Fehlinterpretationen kommen. Bei Dichtern aus den nahe liegenden Ländern ist das, wenn man ihre Sprache kennt, selten der Fall. Aber es kann auch geschehen. Deswegen sind zusätzliche Informationen und gute sprachliche Kenntnisse immer notwendig.

6 Siehe 1, S. 39.

7 Inger Rosengren: Texttheorie. In: Lexikon der Germanistischen Linguistik II. Herausgegeben von Hans Peter Althaus, Helmut Henne, Herbert Ernst Wiegand. Tübingen. Max Niemeyer Verlag 1980, S. 281. 
Ein Übersetzer muß die grundlegenden Kenntnisse des Zeichensystems der Sprache, in welche er übersetzt, kennen. Auch die Möglichkeiten, wie die Inhalte (Gehalte) eines sprachlichen Kunstwerkes geeignet mit Mitteln eines anderen Zeichensystems ausgedrückt werden.

$\mathrm{Da}$ es sich aber in jedem sprachlichen Kunstwerk um besonders organisierte Zeichensysteme handelt, muß auch die ganze Organisation "übersetzt", geeignet übertragen werden.

Die Besonderheiten der künstlerischen Kommunikation lassen sich aber keinesfalls ohne die Annahme erklären, daß sich während der Rezeption der künstlerischen Nachricht zusätzlich zu den allgemein gültigen soziokulturellen Kodes im Rezipienten ein spezieller Kode bildet, der ihn in die Lage versetzt, auch nicht vorkodierte Eigenschaften der Zeichenmaterie und der ebenspezifischen Informationen als Informationsträger zu interpretieren. ${ }^{8}$

Weil aber die Sprache als Zeichensystem menschliche Erkenntnisse vermittelt, muß sie durch den menschlichen Kopf gehen und Verständigungen erreichen. Nicht einmal zwei Menschen verstehen das Vermittelte ganz gleich, so wie es auch beim. Lesen eines Textes größere oder kleinere Unterschiede gibt. Sogar bei vielen alltäglichen pragmatischen Äußerungen oder solchen Texten gibt es geringe Differenzen im Verstehen, da jeder Mensch seine Erfahrungen, seine eigenen Vorstellungen, vor allem aber seine eigenen Gefühlserregungen hat. Wenn es sich um künstlerische Texte handelt, ist eine volle Gleichheit des Verstehens und des Erlebnisses nicht möglich, auch nicht notwendig. Trotzdem müssen wir uns vor totalen Fehlinterpretationen hüten. Dazu verhelfen uns die möglichst exakten Analysen. Text-interne und Text-externe Elemente müssen in Zusammenhänge gebracht und zu Synthesen geführt werden, die zumindest eine Annahme ermöglichen, wenn sie schon nicht ganz überzeugen.

\section{Der Begriff Motto}

Wir gebrauchen das Wort "Motto" oft in bezug auf einen Texttyp, von dem wir kurz, manchmal nur mit einem Wort, das Wesentliche sagen, oder wir geben an, was jemand (auch ein Dichter oder Schriftsteller) gesprochen oder geschrieben hat. Es handelt sich also um das inhaltlich Typische eines Textes, einer Aussage, mag es sich um eine literarische oder sachliche Schilderung handeln. So sagen wir z.B. "Das Motto dieser Schrift ist Nationalhaß." "Sein Motto war, den Menschen nie vertrauen." "Das Motto seines Lebens ist, immer wieder gegen das Unrecht zu kämpfen" usw. 
Ein solches Verstehen des Begriffes "Motto" bedeutet, daß es sich in dem Fall um keine neue Textbildung handelt, obwohl das auch eine kurze Textart (Textsorte) ist. Es handelt sich um etwas, was aus dem Kontext hervorgeht, was wir als eine kurze Charakterisierung, eine kurze Beschreibung mit sachlichen Angaben kennzeichnen können.

Wir können auch von der Tendenz, vom Ziel des Textes sprechen. Die Ausdrücke Leitmotiv und Ténor können da als kurze Angaben des wesentlichen Gehaltes gebraucht werden.

Eine einheitliche Beschreibung, woraus hervorginge, was ein Motto als ein besonderer Texttyp mit eigenen Strukturelementen ist, gibt es nicht. Es ist oft das Wesen des Mottos mit anderen bekannten Begriffen ausgedrückt, meist ist das Ziel, das man mit einem Motto erreichen will, immer klar beschrieben.

In der Literatur gibt es überwiegend Texte ohne ein vorangehendes Motto. Oft ist das Motto explizit oder implizit aus dem Text herauszufinden.

Manchmal kommt der Begriff Motto den Begriffen Leitmotiv, Wahlspruch u.a. nahe.

Jedoch kennen wir trotzdem den häufigen Gebrauch und auch die vielen typischen Kennzeichen, vor allem die Rollen und Ziele des Begriffes "Motto". Es ist möglich, das Motto als einen generellen Texttyp mit einem Kommunikationshorizont der Absicht zu charakterisieren. Aber dieser generelle Texttyp schließt noch andere Texttypen ein. Wenn es sich um selbstständige, neue Textbildungen eines Autors handelt, die der Autor in seinem Werk gebraucht, gibt es bei solchen Bildungen doch einige wesentliche Kennzeichen des Stils dieses Autors. Er wollte nicht nur das literarische Werk für sich selbst sprechen lassen, sondern etwas Zusätzliches bilden. Dann haben wir einen Texttyp, der eigens dem jeweiligen Autor als Ziel entspricht und somit variabel ist. Man kann manchmal auch von einer literarischen Gattung sprechen.

So typisch wie z.B. die Begriffe Roman, Sonett, Epos, Drama u.ä. sind, ist natiirlich der Begriff Motto nicht. Aber auch bei den oben angeführten literarischen Gattungen und Texttypen besteht keine vollkommene Einheitlichkeit.

Es ist in bezug auf den Begriff "Motto" meist wesentlich, daß es aus einem besonderen Grund für einen besonderen Zweck gebildet wird. Dann ist das doch insofern ein Prototyp der Textsorte oder eine literarische Gattung, obwohl variabel.

Schauen wir uns zuerst einige Angaben unter dem Stichwort "Motto" an:

Sehr oft finden wir in den Erklärungen beim Stichwort Motto Leitspruch, Sinngedicht, Wahlspruch. Diese Begriffe können aber nicht ganz als Synonyme des Begriffes Motto aufgefaßt werden.

Es geht oft um einen Text der Mitteilung, wo es also rationale Elemente gibt. Dabei muß es aber auch eine explizite oder implizite Einstellung, ein Gefühl zum Gesagten, zum Inhalt geben. Das breit Ausgesagte in einer Erzählung oder in Gedichtzyklen, in Gedichtbänden kann auch kurz charakterisiert werden, so daß es sich um eine allerkürzeste Zusammenfassung handelt. 
Hier mußte eine Auswahl verschiedener Definitionen getroffen werden, mit denen aber doch eine geeignete Übersicht dargeboten wird:

Wilhelm Wackernagel gebraucht den Ausdruck "Motto" nicht, doch man kann aus anderen Begriffen, die er gebraucht, schließen, daß er daran dachte:

"Unmittelbar an die Betrachtung der Elegie schließen wir die einer anderen Dichtungsart, die mit ihr auf das engste und innigste verbunden ist, die des Epigramms; desjenigen Epigramms nämlich, das den Griechen eigenthümlich ist, das die Römer meistentheils haben bei Seite liegen lassen, das auch die deutsche Literatur erst seit Herder und Goethe kennt, das Epigramm der Empfindung: denn nur dies ist episch-lyrischer Natur." 9

Neuere Erklärungen beim Begriff Epigramm sprechen von einer kurzen Form, meist von zwei Versen (Distichon), aber wir kennen ja viele Epigramme (z.B. von Lessing, Goethe, Grillparzer, K. Kraus u.a.), die länger sind. Verschieden lang ist auch das, was unter dem deutschen Wort Sinnspruch verstanden wird (es handelt sich um einen adäquaten deutschen Ausdruck für den Begriff Epigramm).

Die Wahl eines Zitates aus dem Werk eines anderen Dichters oder Schriftstellers als Motto am Anfang des Buches oder vor jedem Kapitel kennen wir als ein häufiges Stilmittel in der Literatur. Besonders im 19. Jahrhundert, zur Zeit der Romantik und des poetischen Realismus, aber auch schon früher; es war üblich, solche Mottos zu gebrauchen.

Wolfgang Kayser spricht in seinem Buch "Das Sprachliche Kunstwerk" unter dem Begriff Motto nur von dieser Art des Verfahrens bei der Text- und Kontextbildung. So schreibt er:

"Andererseits weist die Verwendung eines dem Kapitel vorgesetzten Mottos darauf, daß die Kapitel als beträchtlich eigengewichtige Teile gemeint sind. In vielen Romanen des 16. und 17. Jahrhunderts enthalten die Mottos das Argument und bekunden damit, daß die Kapitel vom Romangeschehen her als Einheiten konzipiert wurden." 10

Im Buch Poetik in Stichwörtern von Ivo Braak ${ }^{11}$ wird der Begriff Motto nicht als ein besonderes Stichwort erwähnt, sondern es ist unter dem Titel Kurzformen zu finden. Es wird da zuerst der Begriff Sprichwort erwähnt, das der Autor auch Sprechspruch nennt und so erklärt: "... formt volkstümliche - klangliche Mittel (Bsp.: Haus ist kein

9 Wilhelm Wackernagel: Poetik, Rhetorik und Stilistik. Herausgegeben von Ludwig Sieber. Halle a.S. Verlag der Buchhandlung des Waisenhauses 1888, S. 181.

10 Wolfgang Kayser: Das sprachliche Kunstwerk. Eine Einführung in die Literaturwissenschaft. Bern. München. Francke Verlag 1948, S. 178.

11 Ivo Braak: Poetik in Stichwörtern. Literaturwissenschaftliche Grundbegriffe. Eine Einführung. Kiel. Verlag Ferdinand Hirt 1969, S. 150 ff. 
Hase, das läuft nicht weg.). Verwandt mit Sentenz; Weiterentwicklung zum geflügelten Wort, Wahlspruch, Motto...."

Unter den parabolischen Formen hat Braak zwar einen Begriff Spruchgedicht, erklärt es aber als eine Veranschaulichung praktischer Moral. Den Beginn dessen sieht er in der deutschen mittelhochdeutschen Dichtung im. sog. bîspel (Beispielgedicht, Beispielerzählung).

Greifen wir noch einmal ins 19. Jahrhundert zurück und sehen wir uns an, wie der Begriff "Motto" in Wörterbuchern und in den Lexika erklärt wird.

Motto, n. it. (=fr. mot, Wort, ml. muttum, v.l. muttire, mucken, mucksen, leise reden, einen halblauten vereinzelten Ton ausstoßen) der Denk- oder Sinnspruch, Wahlspruch (ohne begleitendes Bild; versch. Devise, s.d.; ein sinnreicher Satz zur Überschrift einer Abhandlung $u$. dgl. ${ }^{12}$

Das ist eine relativ gute, aber ziemlich breite Beschreibung des Begriffes Motto.

Etwas enger ist diese Erklärung:

Motto (ital.) Sinn = oder Denkspruch; Bezeichnung einer einem Schriftsteller entlehnten Stelle, welche eine Schrift zur Andeutung ihres Inhalts oder ihrer Tendenz vorangestellt wird, "Leitspruch, Kennwort". ${ }^{13}$

Ziemlich ausführlich ist das Motto in einigen etymologischen Wörterbuchern erklärt:

Motto, n (-s. PI. -s): Sinn-, Denk-, Wahlspruch. 1717 bei Nehring "Motto, kleines wohlangebrachtes Wort". Aus glbd. ital. motto m., wie frz. mot m. "Wort, Spruch" $u$. span.-port. mote m. "geheimnisvolles Wort", aus mlat. muttum n. "Wort", von lat. mutire, muttire, "mucksen, leise reden", aber mlat. motire "erinnern, erklären". ${ }^{14}$

Im Herkunftswörterbuch des Großen Duden gibt es das Stichwort Motto:

Motto s "Denk-, Wohl-, Leitspruch; Kennwort": Im 18. Jh. aus it. motto "Witzwort; Wahlspruch" entlehnt, das wie entspru. frz. mot "Wort" (s. Bonmot) auf vlat. muttum "Muckser' Wort "zurückgeht. Dies gehört zu einer Reihe von schallnachahmenden Wörtern wie lat. muttire, "mucken, mucksen, halblaut oder kleinlaut reden", lat. mutus "stumm", die elementarverwandt sind mit der unter Maul dargestellten Wortgruppe. ${ }^{15}$

12 Dr. Joh(annes) Christ(ian) Aug(ust) Heyse's allgemeines verdeutschendes und erklärendes Fremdwörterbuch, Sechzehnte einzig rechtmäßige Original-Ausgabe von Prof. Gustav Heyse Hannover. Hahn'sche Buchhandlung 1878, S. 614.

13 Meyers Konversations-Lexikon. Fünfte Auflage. Zwölfter Band Leipzig und Wien. Bibliographisches Institut 1896, S. 569.

14 Deutsches Wörterbuch von Fr. L. K. Weigand. Herausgegeben von Herman Hirt. Zweiter Band L bis Z. Gießen. Verlag von Alfred Töpelmann (vormals J. Ricker) 1910.

15 Duden. Etymologie. Herkunftswörterbuch der deutschen Sprache. Bearbeitet von Günther Drosdovski, Paul Grebe und weiteren Mitarbeitern der Dudenredaktion. Mannheim. Bibliographisches Institut 1963. 
Kürzer ist die Erklärung im Deutschen Wörterbuch Gerhard Wahrigs:

Motto (n. 15) Leitspruch, Wahlspruch; einem Buch od. Kapitel vorangesetzter Spruch od. Ausspruch, der Inhalt od. Ansicht kennzeichnen soll; ein - haben; nach einem -handeln (ital., "Denk-, Leitspruch"). ${ }^{16}$

Ganz kurz erklärt den Begriff Lutz Mackensen in seinem "Deutschen Wörterbuch":

Motto $s(-s ;-s)$ (it., = Wort) Sinn-, Wahlspruch. ${ }^{17}$

Jože Toporišič hat das Wort Motto in seinem Buch Enciklopedija slovenskega jezika nicht. Wohl aber die Begriffe geslo (Stichwort) und geselski članek (Stichwortartikel - alles, was beim Stichwort mit Daten und einer kurzen Erklärung notwendig ist). Jože Toporišič hat das Gedicht Prosto srce ohne Titel in sein Buch Slovenska zvrstna besedila (Slowenische Textgattungen) aufgenommen, und zwar im Kapitel Pesemska besedila (Gedichttexte) mit dem Untertitel Umetne pesmi (Kunstlieder im Unterschied zu Volksliedern). ${ }^{18}$ Das Gedicht wird dort nicht als Motto bezeichnet, das heißt, es ist für Toporišič einfach ein künstlerischer Text. Prešeren hat das Gedicht ursprünglich auch so verfaßt. Aber er hat später gesehen, daß darin die wesentlichen Elemente seiner Lebenserfahrungen, seiner seelischen Lage und seiner Beziehungen zur Welt enthalten, sowie auch der Kern seiner Aussagenuancen und die Perspektive ausgedrückt sind. Er hat deswegen dieses Gedicht als Motto gewählt.

Wir sehen so, daß das Motto zwar nicht als eine besondere Textsorte mit bestimmten formalen Elementen zu definieren ist, sondern in seiner Rolle und Absicht, auf die jeweiligen inhaltlichen Elemente des folgenden Textes vorzubereiten, den Ton vorauszusagen, oder einfach anzusagen, was das Wesentliche in einem Denken, Schreiben, Handeln ist.

Matjaž Kmecl übersetzt in seiner Kleinen Literaturtheorie (Mala literarna teorija) das Wort "Motto" (slov. moto) mit dem angeblichen slowenischen Adäquat geslo.

So heißt es bei Kmecl beim Stichwort MOTTO/GESLO in der Übersetzung: (italienisches Wort, Spruch) - Sprichwort, Vers, Aphorismus, oft Anfiihrung aus bekannten Autoren usw., das vor einem literarischen, manchmal auch vor einem nichtliterarischen Text, auch vor dessen einzelnen Teilen, die Absicht, die Art des Berichtens, den Ton die Perspektive des Textes ansagt. Ein Hilfstitel, ein breiterer Titel, der aber in seiner Aussage freier ist. ${ }^{19}$

16 Gerhard Wahrig: Deutsches Wörterbuch. München. Verlagsgruppe Bertelsmann. Mosaik Verlag GMBH 1986.

17 Lutz Mackensen: Deutsches Wörterbuch. München. Südwest Verlag GMBH CO KG 1986.

18 Slovenska zvrstna besedila. Uredil Jože Toporišič. Sourednik Velimir Gjurin. Ljubljana 1981, S. 281.

19 Matjaž Kmecl: Mala literarna teorija. Ljubljana. Založba Borec 1976, S. 201. 
Kmecl gebraucht in seinem Buch den Begriff noch viermal mit verschiedenen Nuancen und Sichten, die vom Prolog im griechischen Drama bis zu einer Art Refrain in der modernen Dichtung reichen. Das werden wir hier nicht behandeln.

France Verbinc hat in seinem Fremdwörterbuch (Slovar tujk) die folgende Erklärung (in der Übersetzung):

motto $m$ (ital. motto) 1. Stichwort am Anfang des Buches oder Kapitels, gewöhnlich verbunden mit dem Inhalt; 2. kurzer Spruch, Anführung oder Sprichwort mit einer ähnlichen Funktion, schau Epitaph). ${ }^{20}$

Das waren nur einige Angaben, wie das Wort "Motto" als Stichwort, als Begriff mit einer oder mit keiner kurzen Erklärung vorkommt.

Wir werden hier den Begriff als eine Textsorte betrachten, die eine Aussage als eine Art vorangehendes Sujet, eine dem Inhalt des folgenden Textes gedankliche Vorbereitung enthält.

Wenn es sich dabei um mehrere Aussagen handelt, schon mit inhaltlichen Zügen des längeren Textes, kann man wirklich von einer besonderen Textsorte sprechen. Sie variiert im Stil, aber will mit der knappsten Form ihr Ziel erreichen. Ein solches Motto muß inhaltlich (gehaltlich) und sprachlich dementsprechend organisiert und mit dem folgenden Text verbunden sein..

\section{Probleme der Übersetzung eines literarischen Textes}

Probleme einer dichterischen und einer "gelehrten" Übersetzung sind alt. Bei der ersten sollte es sich um eine ebensolche Wirkung auf den Leser handeln wie beim Original, bei der zweiten um eine wortgetreue Übersetzung, daß der Leser den Inhalt und auch die Übersetzung selbst erkennt.

Die Spezifika der Sprachen verlangen Prädikationen ihrer Art, obwohl die außersprachlichen Referenzen gleich sind. Es kommt so auch zu anderen sprachlichen Referenzen. Meinungen, daß beim Übersetzen Kenntnisse der Fremdsprache wichtiger sind, werden durch die entgegengesetzten Meinungen widerlegt.

Bei Prě̌eren gab es verschiedene Situationen, in denen er die Erst- und die Zweitsprache erlernte und gebrauchte. Psychologisch und sozial intimer war das Slowenische. In seiner Muttersprache hatte er früh ein Gefühl für Versifizierungen oder zu vorliterarischen Formulierungen in Versen sowie in Prosaform (vor allem in Briefen) gezeigt. Einige durch Zeugen überlieferte Verse von Prešeren weisen Versformen vom ersten slowenischen Dichter Valentin Vodnik auf. Jedoch hat Prešeren in seiner Jugend Vodniks Muster für eine "niedere stilistische Norm"21 gebracht. Im Brief an die Eltern vom 24. Mai 1924 stellt Boris Paternu einerseits den Einfluß der predigtartigen slowenischen 
literarischen Texte fest, eine Metaphorik, wie man sie auch in solchen Texten findet und die den Bauernleuten nicht fremd war, sogar von ihnen beeinflußt und auch bereichert wurde. Jedoch hat Prešeren das "predigtartige Verfahren und Modell aus der kirchlichen literarischen Norm über die Prosaform in eine individuelle, literarische Norm' versetzt, sich diese ganz angeeignet und sie ganz säkularisiert." 22 Weiter stellt Paternu fest, daß in der Kombination des Bildes und der unmittelbaren Aussage, des Gleichnisses und Schlusses, mit den Junktionen ... plötzlich der Grundriß des späteren Sonetts aufgeht 23

Soziolinguistisch kann man bei Prešeren feststellen, daß er für den intimen sozialen Kreis seiner Verwandten die einheimische slowenische Mundart gebrauchte, deren Kompetenz beim Dichter sehr stark war, was auch die späteren Einflüsse der mundartlichen Lexeme, Idiome, der grammatischen Strukturen in den Gedichten beweisen. Jedoch ist das bei weitem keine Mundartdichtung, wie man sie in der "Modernen Zeit" findet. Prešeren hat seinen reichen, durch die Mundart und durch die bis damals bekannte slowenische Lektüre beeinflußten Idiolekt zu einer literarisch hochrangigen Stufe entwickelt. Eine einheitliche Standardsprache hatten die Slowenen damals noch nicht. Prešeren hat zu ihrer Entwicklung viel beigetragen, besonders was die Bildhaftigkeit und die damit verbundenen intellektuellen Elemente betrifft. Etliche Briefe, besonders ein Brief an den tschechischen Dichter F.L. Čalakovsky vom 10. März 1833 beweisen, daß Prešeren um die letzteren sehr bemüht war.

Ein Ringen mit dem ihm bekannten Wortschatz und eine Furcht, daß gewisse Wörter für die slowenische Standardsprache, die im Entstehen war, nicht geeignet oder einem Fremden nicht verständlich wären, beweisen auch Prešerens deutsche Übersetzungen einiger slowenischer Wörter in Klammern. Gewiß wollte er aber auch Čelakovsky, der ja der deutschen Sprache vollends mächtig war (so wie Prešeren) die Aussagen klarer machen.

So sehen einige Syntagmen und Begriffe im Brief aus: gorkiga (warmen) zagovarjovca ... ljudske pesmi (Volkslieder) ... tercjali (Bettbrüder) ... V rokopismih (Handschriften).

Prešerens Übersetzungswillen und -vermögen sowie einige Schwierigkeiten wegen der noch fehlenden gut ausgearbeiteten slowenischen Standardsprache erkennt man also auch in diesem Brief. Ebenso sind darin Prešerens Sprachgefühl und Sprachkenntnisse in beiden Sprachen gut ersichtlich. Alle im Brief ins Deutsche übersetzte Wörter gibt es heute in der slowenischen Standardsprache, nur mit geringeren phonematischen und morphematischen Veränderungen. Maks Pleteršnik hat in seinem Slowenisch-deutschen Wörterbuch (Slovensko-nemški slovar 1894, Reproduzierter

21 Boris Paternu: France Prešeren in njegovo pesniško delo. Ljubljana. Mladinska knjiga 1976, S. 46.

22 Ebenda, S. 46.

23 Ebenda, S. 47. 
Nachdruck 1974) beim Adjektiv górek drei Bedeutungen angeführt: 1) warm, 2) gram sein, 3) ein Synonym für grenek (bitter). Auch im Sinne "erhitzt", "derb" gibt Pleteršnik die Bedeutung an. Beim Substantiv gorkôta erwähnt er die Bedeutung Wärme und nennt das Derivat gorečnost in der Bedeutung Eifer. Es würde auch bei Prešeren die Übersetzung gorkiga mit so eifrigen besser sein, aber die erste Übersetzung schien Prešeren natürlicher zu klingen, weil das Wort gorki, gorko in der Bedeutung "warm" geläufiger war.

Die Benennung ljudske pesmi ist heute auch der slowenische Fachausdruck für Volkslieder.

Das Wort tercjal gibt es in der modernen slow. Standardsprache mit dem Suffix tercijalec (P1. tercijalci); die deutsche Bedeutung "Betbruder, Frömmler". Die Handschrift heißt im heutigen Standardslowenischen rokopis.

Prešeren baute in die deutsche Übersetzung des hier behandelten Mottos und ursprünglich selbständigen Gedichtes intellektuelle Elemente ein, wollte aber nicht das Original und den Texttyp verletzen. Er wählte in der Übersetzung keine Wiederholung, sondern eine Art Deutung. So klingt das Original unmittelbarer, intimer, die Übersetzung hat etwas mehr gedankliche Elemente, die teils ganz kurze Analysen des Originals sind.

Einen relativ präzisen Ausgleich der dichterischen Aussage erreicht Prešeren mit den reziprok gewechselten Substantiven (alles Abstrakta) und Verben, was die Stellung und die Expressivität betrifft. Je zwei Substantive, je zwei Adjektive und je zwei Verben gehören zu den zentralen sprachlichen Referenzmitteln, mit denen die expressiven und gedanklich ausgeglichenen Prädikationen gebildet werden. Das entspricht auch dem Texttyp (dem literarischen Begriff) "Motto".

Wir können einer allgemeinen Ansicht zustimmen, daß "der literarische Text als etwas Ganzheitliches Zeichen besitzt, schon etwas äußerlich Erkennbares, eine Ordnung oder Gliederung in sich, die das Produkt bestimmter Abhängigkeiten zwischen größer oder kleiner bemessenen Segmenten ist. Laute, Formen, Wörter, Sätze fungieren nicht außerhalb und unabhängig vom Bestimmungsganzen des Textes, sondern immer nur in bezug auf die Mitteilung, die er transportiert, bzw. auf den Sinn, den er hervorrufen soll." 24

Bei der Übersetzung werden meist schwierige Voraussetzungen verlangt. Es gibt da Kontradiktionen, man kann aber auch eine Übersetzungsart wählen, verfolgen. Schauen wir uns einige von den Voraussetzungen an:

- Die Übersetzung soll den Sinn, den ganzen Sinn und nichts als den Sinn wiedergeben.

- Sie soll vom Sinn "in sehr hohem Maß" absehen und nach Wörtlichkeit streben.

24 Gothard Lerchner: Sprachform von Dichtung. Linguistische Untersuchungen zu Funktion und Wirkung literarischer Texte. Berlin und Weimar. Aufbau - Verlag 1986, S. 37. 
- Die Übersetzung soll das Erlebnis des Originals vermitteln.

- Sie soll dessen Kenntnis vermitteln.

- Die Übersetzung soll auf den Leser dieselbe Wirkung wie das Original ausüben, auch wenn sie dann anders aussehen muß.

- Sie soll aussehen wie das Original, auch wenn die Wirkung auf den Leser eine andere ist.

- Die Übersetzung soll sprachlich der Zeit des Originals angehören.

- Sie soll sprachlich der Zeit des Übersetzers angehören.

- Die Übersetzung soll sich lesen wie ein Original, weil sich das Original als ein solches liest; weil Abweichungen vom einheimischen Sprachgebrauch den Leser bloß befremden; weil eine Überfremdung der Muttersprache dem Sprachgefühl des Lesers schadet; weil sich für Wörter und Wendungen der einen Sprache in der anderen meist etwas Entsprechendes findet;

- Die Übersetzung soll als solche erkennbar sein, weil der Gedanke an die sprachliche Form gebunden und nur mit dieser übertragbar ist; weil deshalb vom Leser zu verlangen ist, daß er sich eine besondere Übersetzersprache aneignet;

weil dadurch die Ausdrucksfähigkeit der eigenen Sprache erweitert wird; weil die Wörter und Wendungen in zwei Sprachen sich nie ganz decken. ${ }^{25}$

Eine Übersetzung kann nie wie ein Original wirken. Eine Annäherung ist jedoch möglich. Aber die Übersetzung ist ein anderes Sprachgebilde, insofern ist sie auch immer ein neues Erlebnis und gibt schon wegen der Sprache neue kognitive Elemente.

\section{Die Wahl des Mottos und die individuellen Kennzeichen der Übersetzung bei Prešeren}

Prešeren hat das vierzeilige Gedicht Prosto srce, daß er als Motto für seinen Gedichtband 1846 wählte, schon am 26. Mai 1838 im Illyrischen Blatt (Beilage der Laibacher Zeitung) als ein selbständiges Gedicht Prosto srce mit der eigenen deutschen Übersetzung Das freie Herz veröffentlicht. Es ist eine Art Abschluß der Gedichte, die 
Prešeren von 1831 - 1838 im Illyrischen Blatt veröffentlicht hat und die hauptsächlich an Julija Primic, seine große unerhörte Liebe gerichtet waren.

Nach Anton Slodnjak sollte Prešeren etliche seiner Übersetzungen der slowenischen Gedichte oder überhaupt nur im Deutschen verfaßte Gedichte geschrieben haben, weil Julija die slowenische Sprache nicht mochte. Sie sprach fast nur deutsch, wollte nach Angaben Slodnjaks und nach den Äußerungen Anna Jelovšeks (Prešerens uneheliche Lebensgefährtin) mit Leuten, die der deutschen Sprache nicht mächtig waren, einfach nicht sprechen.

Das mag bei Prešeren wirklich ein Grund für die deutsch verfaßten und für die ins Deutsche übersetzten Gedichte gewesen sein. Gewiß ist aber das nicht der einzige Grund.

Prešeren ist schon früh zweisprachig geworden, er las viel in deutscher Sprache, studierte hauptsächlich in deutscher Sprache, korrespondierte in ihr als gebildeter Mann. Freude an dieser Sprache ist ihm anerzogen worden, er bekam Sinn und Freude für die deutsche Sprache auch durch Anregungen in literarischen und überhaupt humanistischen Kreisen.

Trotz der Traumata, die er und das ganze slowenische Volk seitens der deutschsprachigen Österreicher erleiden mußten, erfaßte und fühlte Prešeren auch den Wert der deutschen Sprache. Er schätzte und gebrauchte sie, obwohl er das Leiden der Geringschätzung und Unterdrückung der slowenischen Sprache nicht hinnehmen und nicht verkraften konnte.

Seine Einstellungen zum Dichten in der deutschen Sprache drückte Prešeren selbst in einigen deutsch verfaßten Gedichten im Zyklus Des Sängers Klage aus.

Für das längste und künstlerisch gelungenste deutsche Gedicht Dem Andenken Mathias Čop, das Prešeren in Terzinen verfaßte, kann man wirklich sagen, daß die einmalig gelungene deutsche Fassung Prešeren und dem slowenischen Volke dazu dienen sollte, den großen Gelehrten Mathias Čop und die damaligen historischen und geistigen Verhältnisse im Land der Slowenen und in Alt-Österreich zu würdigen sowie in Europa überhaupt dichterisch in einer klassischen Form mit großer Aussagekraft und mit einem weiten Horizont darzulegen.

Obwohl das Gedicht in deutscher Sprache verfaßt ist, ist es dem Inhalt nach das slowenischste von allen Gedichten France Prešerens.

Anton Slodnjak sagt, daß die Elegie neben der ästhetischen Schönheit auch wichtige Daten von Prešerens schöpferischer Kraft gibt und daß wir berechtigt seien, zu schließen, daß der Dichter die Elegie in kürzester Zeit, sozusagen "im Handumdrehen" geschrieben habe. Das sollte uns nach Slodnjak zum Staunen bringen, da es sich doch bei Prešeren um eine Fremdsprache (Zweitsprache) handle. ${ }^{26}$ 
Prešeren war koordiniert zweisprachig, und zwar ohne Diglossie. Wenn bisweilen etwas von der Diglossie zu spüren ist, so handelt es sich nicht um die sprachliche Kompetenz als solche, sondern um die Beziehungen zu soziokulturellen Kreisen, zur allgemeinen kulturellen Umgebung, wo die Slowenen damals keine glückliche, gleichberechtigte Lage hatten.

Was das hier behandelte Gedicht (das spätere Motto) und die Übersetzung betrifft, handelt es sich im Original und in der Übersetzung um einige Aussagen, wo Mitteilungen und Gefühle des intimen Lebens enthalten sind. Man kann nur die künstlerische Ebene fühlen, das heißt, nur den Eindruck mit dem Wesen der wichtigsten Äußerungen berücksichtigen.

Dieses Wesen ist:

Unglück und Leiden, Resignation, wieder Sehnsucht, einen gehobenen Zustand zu erreichen, der das Leben trotz des Leidens bereichert.

\section{Prosto srce}

Sem dolgo upal in se bal, slovo sem upu dal;

srce je prazno, srečno ni, nazaj si up in strah želi.

Wir werden hier zuerst für die nur deutschsprachigen Leser noch eine zusätzliche wörtliche Übersetzung nachtragen, d.h., ohne den einen oder den anderen dichterischen Stil zu berücksigtigen, nur daß gezeigt wird, wie die Aussagestruktur in Sätzen aussieht und daß dann aus dieser Sicht auch die dichterischen Texte im Vergleich und mit Analysen besser verstanden werden können:

Ich habe lange gehofft und mich gefürchtet, ich habe der Hoffnung, der Furcht den Abschied gegeben;

das Herz ist leer, ist nicht glücklich, es wünscht sich die Hoffnung und die Furcht zurück.

Prešerens dichterische Übersetzung:

Das freie Herz

Am Herzen hat Hoffnung, hat Furcht genagt;

nun hab'ich beiden Lebewohl gesagt.

Das Herz ist frei, auch ruhig, glücklich? Nein.

Ach, zögen doch Hoffnung und Furcht wieder ein! $!^{27}$

Es handelt sich doch um Mitteilungen, und die kann man syntaktisch zergliedern: fünf affirmative Sätze, Hauptsätze, Asyndeton, vier Mitteilungen, komplexe Mitteilung. 
Man kann im Slowenischen alle fünf Sätze als eine kopulative Parataxe mit der Konjunktion und auffassen, oder es sieht das komplexe Satzgebilde in der Analyse so aus:

Im Slowenischen sind die ersten drei Sätze ähnlich gegliedert.

\section{Sem dolgo upal in se bal,}

Zwei Aussagesätze, der zweite elliptisch zato(deswegen), in(und), toda(aber)

toda(aber), zato(deswegen), in(und)

zato (deswegen)
Ich habe lange gehofft und mich gefürchtet, slovo sem upu, strahu dal;

ich habe der Hoffnung, der Furcht den Abschied gegeben; srce je prazno, toda (jedoch/aber), srečno ni, das Herz ist leer, (jedoch/aber) ist nicht glücklich, nazaj si up in strah želi.

es wünscht sich die Hoffnung und die Furcht zurück.

Nach der Konjunktion deswegen wäre in den deutschen Sätzen die Wortfolge anders.

deswegen/und/aber und/deswegen/aber und/deswegen
Am Herzen hat Hoffnung, hat Furcht genagt; nun hab' ich beiden Lebewohl gesagt.

Das Herz ist ruhig und/aber auch glücklich? // Nein. Ach zögen doch Hoffnung und Furcht wieder ein!

Im Deutschen kann man die Struktureinheiten auch glücklich? Nein. als elliptische Sätze betrachten. So bekommen wir sechs Sätze. Auch hier wäre nach der Konjunktion deswegen die Wortfolge anders.

Das inhaltliche Satzverhältnis bleibt jedoch in jeder Fassung (im Original, in der wörtlichen Übersetzung) ganz gleich.

affirmativ

konsekutiv, adversativ

konsekutiv, adversativ $\longrightarrow$ adversativ konsekutiv

Im Slowenischen könnten vier Verse, der dritte mit zwei Sätzen, selbständig stehen. Im vierten müßte nur das ausgelassene Subjekt erwähnt werden: srce ...

Im Deutschen ist die Gliederung trotzdem etwas komplizierter. Es müßten im zweiten Vers, der ein Pronomen als Substitut für zwei Dativobjekte hat, die beiden Subjekte des ersten Satzes stehen (Hoffnung, Furcht). Prešeren gebraucht beide wieder in der letzten Zeile. Das heißt, daß er die beiden Abstrakta als einen Begriff auffaßte, der auch mit dem Abstraktum trpljenje (Leiden) ersetzt werden könnte. Die letzten beiden Verse könnten als ein komplexer Satz (Gesamtsatz) allein stehen, könnten eine selbständige komplexe Aussage sein.

Zwischen den ersten zwei Versen kann es zu einer Zäsur kommen; dann entsteht zu den nächsten beiden Sätzen klarer ein konsekutives und ein adversatives Verhältnis. Auch zwischen dem dritten und dem vierten Vers kann man ein kopulatives und ein 
konsekutives Verhältnis annehmen. Eine Folge ist immer mit einer Ursache verbunden, beides ist eine logische Einheit.

Das komplexe inhaltliche Verhältnis ist in der deutschen Übersetzung im syntaktischen Bau zwar wirklich sehr ähnlich wie im slowenischen Gedicht, aber nur bis zum dritten Satz. Auch da könnte man zwischen dem zweiten und dritten Satz von einem kopulativen oder von einem konsekutiven Verhältnis sprechen. Aber dann kommt plötzlich die eingeschobene elliptische Frage und die kurze Verneinung mit der Abtönungspartikel: Auch ruhig glücklich? Nein.

Der letzte Satz ist dann wieder eine Folge, die aber formal nicht mit einer Konjunktion der konsekutiven Parataxe eingeleitet wird, sondern mit einer Interjektion einer Partikel. Diese und die beiden kurzen Bildungen (Frage mit Ellipse, Verneinung mit der Abtönungspartikel) zerstören zwar ein wenig den Rhythmus, wenn man das Gedicht rezitiert, den gedanklichen Rhythmus beleben sie aber. Es ist eine plötzliche kurze gedankliche Gliederung eingeschoben. Dieses rhetorische Mittel spricht von der intellektuellen Rolle der deutschen Sprache beim Dichter. Er hatte ja die Schulbildung in deutscher Sprache erhalten, Slowenisch gab es bei ihm als Unterrichtsfach. Das Studium der Rechtswissenschaften absolvierte er auch nur in deutscher Sprache. Man spürt etwas vom juristischen Hauch in der plötzlichen Frage und in der kurzen Antwort. Es ist aber sofort wieder die Gefühlsregung da, mit einer Interjektion eingeleitet, die den tiefsten Wunsch bekräftigt.

Im ersten und zweiten Vers gibt es in der deutschen Übersetzung außer dem Hilfsverb sem (habe) keine einzige Wortart des slowenischen Originals. Das ist eine Übertragung in eine Satzstruktur mit einer neuen Wortwahl und Wortstellung. Man könnte das eine Umschreibung, sogar eine Deutung nennen. Das Wort Lebewohl als ein Abschiedsgruß (eine Grußformel), aber nicht direkt in einer Kommunikation gebraucht, ragt ein wenig aus dem Kontext.

Vielleich hat der Dichter im dritten Vers auch deswegen das slowenische Wort prazno (leer) mit dem deutschen Wort frei übersetzt. Dieses klingt im ersten Moment als eine größere Entlastung, aber nach der adversativen Satzstruktur mit zwei elliptischen Fragesätzen, wo die beiden Prädikatsadjektive ruhig, glücklich und die sofortige kurze Antwort Nein folgen, was alles vom seelischen Ringen zeugt, wird das Wort frei schon vor der Verneinung negiert. Das klingende Prädikatsadjektiv frei ist also nicht die Feststellung einer solchen Lage, daß der Dichter alles vergessen hätte und nun glücklich wäre, sondern es schließt sich der ganze komplexe Satz mit seinen Aussagen dem slowenischen Wort prazno adäquat (leer - in dem Fall nicht glücklich leer) an, was sofort mit dem adversativen Satz bestätigt wird.

Auffallend ist im letzten Satz der Konjunktiv, der unmittelbar der Interjektion folgt. Einerseits spielt er die Rolle des akustischen Eindruckes mit dem langen $\ddot{o}_{,}$anderseits ist damit die sprachliche Information, die im Slowenischen mit dem Verb želi im Indikativ (wünscht) ausgedrückt ist, zustande gekommen. Es wäre im Deutschen un- 
möglich, dieselbe sprachliche Struktur zu bilden, weil dann die Silbenzahl und der Rhythmus, das Metrum im Vergleich mit dem Original noch zerhackter wirken würden. Das Wort Hoffnung hat gegenüber dem slowenischen Adäquat up drei Silben. Auch das Syntagma nazaj si želi ist im Vergleich mit dem entsprechenden deutschen Syntagma, wenn dieses gebraucht worden wäre (wünscht sich zurück), kürzer, was die Laute betrifft $11: 13$.

Gewiß ist aber der Gebrauch des Konjunktivs in diesem deutschen Vers bei Prešeren auch eine Sache des intellektuellen Bereiches, weil der Konjunktiv im Deutschen von den einfachen Leuten im süddeutschen Sprachraum nicht so oft gebraucht wird.

Im Slowenischen gibt es den Konjunktiv als eine besondere grammatische Form nicht, die sprachlichen Mittel für den Ausdruck einer Möglichkeit, eines Wunsches, einer Bedingung, eines Zweifelns im weitesten Sinne sind anders. Prešeren hat im slowenischen Gedicht ein reflexives Verb als ein semantisches Mittel gewählt (želi si = wünscht sich), in der deutschen Übersetzung steht dafür ein grammatisches Mittel des Modus. Solche Mittel hat er in der Schule erworben.

Überwiegende Ausdrucksweisen:

Prosto srce

1. upal se bal verbal

habe gehofft habe mich gefürchtet

2. slovo upu strahu nominal

der Hoffnung der Furcht Abschied gegeben

3. srce je $\rightarrow n i \rightarrow \quad$ verbal (Prädikatsadjektive)

das Herz ist ist nicht

4. up strah si želi nominal

die Hoffnung die Furcht

Das freie Herz

1. am Herzen Hoffnung Furcht nominal

2. hab'ich Lebewohl ("lebe wohl") gesagt verbal

3. das Herz ist $\rightarrow$ Nein $\rightarrow \quad$ verbal (Prädikatsadjektive)

4. zögen Hoffnung Furcht ein nominal

Wir sehen da die gleiche Zahl von Sätzen mit überwiegend nominaler und überwiegend verbaler Ausdrucksweise, jedoch sind sie in einer anderen Reihenfolge gegliedert. Die typischen Vorgänge und die typischen Zustände (seelische Vorgänge) werden in den beiden ersten Zeilen wechselseitig verbal-nominal, nominal-verbal ausgedrückt, in den beiden letzten bleibt aber die gleiche Reihenfolge. Im letzten Vers ragen die beiden konträren seelischen Zustände wie Antonyme heraus. Damit bekommen sie 
eine sehr starke inhaltliche Betonung, werden als die wesentlichen Elemente der Aussage noch besonders deutlich.

Es können zwar kleine Abweichungen im Metrum und Rhythmus bei den einzelnen Lesern vorkommen. Im Original ist das kaum möglich. Etwas mehr Möglichkeiten gibt es in der Übersetzung. Aber auch da nicht allzuviele. Der Abschiedsgruß (hier nominal - das Lebewohl oder verbal - "Lebe wohl" empfunden) kann wegen einer starken adversativen Einleitung auch stärker, vielleicht besonders stark betont werden. Auch das vorangehende Hilfsverb hab und das Zahladjektiv beiden können einen stärkeren dynamischen Akzent tragen, damit kommt nämlich der plötzliche Gegensatz, der starke Entschluß ganz besonders deutlich zum Ausdruck. Die Ikten müssen besonders stark in den Wörtern frei, ruhig, Nein! gefühlt und ausgedrückt werden. Das Ganze wirkt wie ein tiefes Stöhnen, ein gedämpftes Aufschreien.

Der letzte Vers ist rhythmisch und metrisch gleichmäßiger; als wäre der Dichter nach dem dramatischen Abbrechen mit den ständig sich wechselnden Gefühlen der Liebe, der Hoffnung, der Angst zur Einsicht gelangt, daß es doch besser ist zu fühlen als entleert zu sein. Er drückt nun diese reife Erkenntnis mit klaren, bedachten, sich aussöhnenden Gefühlen und Gedanken aus. Der slowenische Literaturwissenschaftler Anton Slodnjak, ein Prešeren-Spezialist, charakterisiert Prešerens deutsche Übersetzung dieses Gedichtes (Mottos) mit den folgenden Bemerkungen: "Im Vergleich mit der elegischen Melodie des Originals ist die Übersetzung dramatischer und unruhiger bewegt." 28

\section{Schlußbemerkungen}

Die deutsche Übersetzung wirkt intellektueller, das Original ist natürlicher, unmittelbarer, geläufiger.

Die Begriffe natürlich und intellektuell sind aber keine Werturteile, bedeuten auch nicht immer etwas Einfaches, Primitives und etwas Höheres; in der Kunst schon gar nicht. In einem Motto wie dieses, wo es gedankliche und gefühlsmäßige Äußerungen gibt, kommt es bei der Übersetzung, die immer zusätzliche gedankliche Anforderungen verlangt, zu vielen wechselseitigen Wirkungen.

Wenn man die sozio- und psycholinguistisch anders erworbenen Sprachen bei vielen Menschen erforscht, spürt man sehr oft unterschiedliche Kompetenzen in der Alltagssprache. $\mathrm{Zu}$ einem kommunikativen Schaden kann es aber nur kommen, wenn ein Partner eine starke Diglossie mit Restringierungen in einer Sprache aufweist, besonders wenn beide Partner durch verschiedene Vorurteile geprägt sind.

28 France Prešeren. Pesmi in pisma. Redigiert von Anton Slodnjak. Ljubljana. Mladinska knjiga 1960, S. 279 . 
Ein viel schlechteres Beherrschen der Zielsprache kann zu schlechten oder falschen Übersetzungen führen. Aber es ist beim Übersetzen sogar wichtiger, daß man die Muttersprache gut beherrscht.

Prešeren hat beide Sprachen hervorragend beherrscht, wie das alle seine Schriften und seine Biographie beweisen. Auf eine besondere Art auch dieses Motto.

Auch der Leser muß beide Sprachen gut kennen, dazu noch die historischen und die sozialen Umstände, aus denen die sprachlichen Kompetenzen und Handlungen zu verstehen und zu bewerten sind. Im Kunstwerk muß der Leser, wenn er das Werk besser verstehen und erleben will, auch den individuellen künstlerischen Stil, der aus vielen Werken (Gedichten) eines Autors zu ergründen ist, gut kennen.

Die Rezeption eines Kunstwerkes ist zwar bei jedem Leser anders, aber pauschale Werturteile überzeugen nicht. Wissenschaftliche Analysen vermeiden sie.

Wir haben die sprachliche Charakterisierung der deutschen Fassung des hier behandelten Mottos von Prešeren, wie sie der brillant zweisprachige große Literaturwissenschaftler Anton Slodnjak ausgedrückt hat, schon erwähnt. Slodnjaks Kennzeichnung kann man auch als eine Rezeption betrachten.

Umfangreicher, mit vielen analytischen philologischen Details, hat diese Studie einen Vergleich zwischen den beiden Texten zu ziehen versucht.

Unterschiede gibt es trotz Prešerns koordinierter Zweisprachigkeit ohne Diglossie im sprachlichen künstlerischen Ausdruck dieses Mottos doch. Einige psychische und soziale sowie intellektuelle Einflüsse auf die sprachlichen Äußerungen in der Kompetenz von Prešeren sind auch in den künstlerischen Werken ersichtlich, ohne daß der künstlerische Wert verlorenginge.

Eine Art Diglossie zwischen den beiden Sprachen zeigt Prešeren damit, daß er in der deutschen Fassung eine schärfere intellektuelle Gliederung gewählt hat. Aber die psychische, gesellschaftliche, materielle Lage Prešerens war ja bei seiner großen Liebe und bei allen Hoffnungen nicht nur vom Gefühl geprägt, sondern auch eine objektive soziale Wahrheit, was analytische-intellektuelle Elemente auslöste. Das wird aber nicht direkt ausgedrückt, sondern mit den Schilderungen der seelischen Vorgänge, in der die objektiven Verhältnisse mitklingen. Von diesen Verhältnissen und von den vielen Ausbreitungen und Details der damit verbundenen Gefühle erfährt der Leser in den folgenden Gedichten des ganzen Bandes, dessen Motto das hier behandelte Gedicht ist.

Von der Übersetzung hört man bisweilen, daß das Wort genagt zu prosaisch klingt. Aber diese Kritik kann man auch mit der These von den stärker ausgedrückten dramatischen Elementen widerlegen.

Auch das Wort Lebewohl klingt wie ein unmittelbarer Dialog in einer dramatischen Situation. Dann grübelt der Dichter, ringt mit sich selbst, er kommt zu einem Entschluß, ergibt sich nicht in der Entleerung, gerät nicht in eine Resignation, sondern trachtet zumindest nach einer neuen Aktivität, nach der Veränderung der Lage. Auch das ist etwas Intellektuelles und Dramatisches. 
Wie dem Dichter diese Veränderung gelang oder mißlang, wissen wir aus seiner Biographie. Jedenfalls geriet er innerlich und äußerlich in eine schwierige Lage, wollte aber im geistigen und psychischen Bereich sowie sonst im Leben noch sehr aktiv bleiben. Es ist ein Entschluß, für die seelischen Aktivitäten noch zu ringen, ob es gelingt oder nicht. Kein gänzlicher Zusammenbruch oder Tod sind schon da. Man lebt weiter, mit viel Verlust, doch mit der Hoffnung, wieder hoffen zu können, wieder etwas vom volleren Leben in sich zu wecken, einfach weiterzuleben.

Jedenfalls bezieht das alles auch etwas mehr gedankliche Elemente in den deutschen Aussagebau ein.

Der slowenische Aussagebau enthält bei Prešeren lexikalisch denotativ und konnotativ Semanteme aus der allgemeinen europäischen und aus der slowenischen volkstümlichen Kultur, die ein Teil der europäischen im alpinen und subalpinen Raum ist, aber so, daß der Dichter seine individuell geprägte Metaphorik schuf und den eigenartigen Stil fand.

Das Original dieses Mottos ist wirklich elegischer, zarter. Als würde der Dichter immer wieder in die Sprache seiner Jugend mit den Gefühlen der frühen Liebe, mit dem Klang der dörflich gehobenen Sprache, die ihn mit der schönen Natur verband, wo Menschen lebten, mit denen er intimer fühlte, wo es für den Dichter noch keine größeren sozialen, intellektuellen Konflikte gab. Das spätere intellektuell reiche Leben bereicherte aber diese elementare Ausdruckskraft.

Musikalischer sind die slowenischen Verse, der Dichter hat ja die Muttersprache auch mit einem elementaren Klang, in Verbundenheit mit dem Heimatort und der herrlichen Umgebung (die er oft besungen hat), mit den nächsten Verwandten, mit der Mutter empfunden.

Es war aber nicht nur Melodie, auch Erwägungen als gedankliche Elemente gab es immer auch auf dem Lande, in der Familie Prešeren. Auf den ersten Blick waren sie kärglicher, aber desto unmittelbarer, prägnanter, trefflich bildhaft auch in alltäglichen, jedoch immer intensiv erlebten innerlichen und äußerlichen Situationen. Das alles spürt und erfaßt man im Original dieses Gedichtes und in vielen anderen slowenischen Gedichten Prešerens.

Gespräche mit Einschiebseln von Erwägungen, mit Fragen und Antworten, mit Schlüssen kennen auch die einfachen Leute. Oft werden dafür Sprüche und Textteile, auch Gedichte verwendet, etliche aus dem Volksmund, aus der religiösen und weltlichen Lektüre übernommen. Auch spontane selbständige, immer wieder neu geschaffene sprachliche Äußerungen gab es und gibt es auf dem Lande. Zur Zeit Prešerens war das geistige Leben auf dem Lande noch viel reger. Die meisten slowenischen Intellektuellen kamen vom Lande.

All das spürt man im Original dieses Mottos und in der Übersetzung von Prešeren, als Folge der intensiven Erlebnisse, der Belesenheit und der Reife seiner damaligen Al- 
tersstufe. Besonders in der Übersetzung bemerkt man die Belesenheit des Dichters, das sprachliche Kommunizieren mit Intellektuellen in deutscher Sprache.

Die deutsche Fassung des Mottos muß man wirklich als eine Übertragung betrachten. Nicht nur in sprachlicher Hinsicht, sondern auch in einer komplexen situativen Hinsicht. Es handelt sich gleichzeitig um eine Adäquanz und um ein Komplement. Eine Einheit in Parallelen, die von einem reichen Kern ausgehen und konvergent zu einem Kern führen.

\section{Povzetek \\ PREŠERNOV LASTNI PREVOD KASNEJŠEGA MOTA PROSTO SRCE (DAS FREIE HERZ). MATERINŠČINA IN DRUGI JEZIK V PESNIŠKEM KONTEKSTU}

Pesniški jezik je vedno grajen iz strukturalnih prvin jezika. Res gre za posebno organizacijo jezika in za različne konotacije, vendar so $v$ vseh jezikovnih variantah in zvrsteh v taki ali drugačni meri ter obliki izražene čustvene, intelektualne in retorične prvine. Pri ustvarjanju besedila je treba graditi enote hierarhične stopnje v dimenzijah jezikovnega zaporedja. Tudi vzporednost igra pomembno vlogo.

Čeprav je težko vzdržati popolnoma enotne strategije za različne besedilne zvrsti, je vendarle možno najti besedila, ki jih lahko razvrstimo po tipologiji.

Pri pojmu moto najdemo v virih včasih enačenja s pojmi vodilni motiv, volilo, epigram, sentenca, geslo ipd. Vsekakor pa gre pri motu, pa najsi ga obravnavamo kot posebno besedilno zvrst ali ne, za besedilni tip $\mathrm{s}$ komunikacijskim horizontom namena. Vsebuje kratko sporočilo, stališče, čustvo, kar naj vnaprej karakterizira besedilo (prozno ali pesniško), ki sledi.

Za vsako jezikovno komunikacijo, za recepcijo literarnega besedila še posebej, velja, da mora komunikacijski partner poznati pravila (jezikovni sistem) jezika, v katerem piše pesnik ali pisatelj, nekatere zunajjezikovne dejavnike iz socialnih in psiholoških položajev pesnika in (če obstajajo) oseb, o katerih piše, tudi odnose do sveta in moralne kodekse pa še nekaj drugih življenjskih podatkov o pesniku. Vse to mora recipient poznati, če hoče dobro razumeti literarno delo. V umetniški komunikaciji pa se seveda lahko ob recepciji v bralcu pojavijo specialni kodi, ki omogočijo interpretiranje sistema znakov, ki niso vnaprej kodirani. Vendar je vedno treba obvladati temeljni sistem znakov, pri prevajanju dva sistema znakov.

Pri prevajanju pesmi obstaja že od davnega dilema, ali naj prevajamo tako, da dobimo kar najbolj verno besedno in stavčno zvezo, se pravi, da bralec takoj spozna izvirnik (to imenujejo nekateri strokovnjaki "učeni prevoc"), ali pa prepesnimo pesem tako, da dobimo ton in sugestivno estetsko moč izvirnika tudi z drugačnimi jezikovnimi strukturami (to imenujejo "pesniški prevod").

Prešeren je uporabil v svojem prevodu pesmi (mota) Prosto srce oba principa, vendar daleč prevladuje pesniški princip. Pri natančni analizi opazimo, da obstaja tudi v prevodu miselno-izpovedni sistem izvirnika, ki se kaže v menjavanju prevladujočega samostalniškega ali glagolskega izražanja, kjer so zajeti vsi temeljni pojmi pesnikovega duševnega stanja, povezani s socialnim, čustvenim in intelektualnim ozadjem $v$ odnosu do Julije. Prejšnje in novo duševno stanje kaže v opozicionalnem zaporedju še vedno dinamiko, željo po duševno bogatem življenju. V vsem besedilu je čutiti kljub mirnim tonom verzov določeno napetost, tudi dramatičnost. Več slednje začutimo v nemškem prevodu, medtem ko je v izvirniku več elegičnosti. Prevod ima več ostrih cesur, modalnih členkov, ki ustvarijo bolj razsekan ritem, dajejo več miselnih ostrin in dramatičnosti. Tudi v dialoškem smislu izraženo slovo od preteklega trpljenja (čeprav ne direktno $v$ dialogu), dve vprašanji in ostro zanikanje dajejo prevodu več dramatičnosti. Zadnji verz pa kaže $z$ uporabo nemškega glagola v konjunktivu, ki ima posebno zvočen korenski samoglasnik in ki mu je Prešeren dodal še medmet $A c h$, posebno žalost in globoko željo po vrnitvi bolj polnega duševnega življenja. Tako deluje prevod kot nekakšen komplement ali paralela izvirnika $\mathrm{z}$ istim izhodiščnim jedrom in konvergenco $v$ isto jedro. Jezikovno je pri tem opaziti bolj tekoč, naravno občuten, na prvotno intimno socialno in zemljepisno okolje vezan jezik izvirnika. Pri tem sta lepo vidna vloga in moč 
materinščine (jezika prve socializacije). Zametke umetniškega izražanja v materinščini najdemo pri Prešernu že pred objavo pesmi. Po B. Paternuju jih kaže tudi pismo staršem iz l. 1924. V vsem se zrcali tudi Prešernov stik z dotedanjo slovensko književnostjo.

Nemški jezik je bil Prešernu jezik študija in širših komunikacij v srednjeevropskem prostoru, tudi komunikacij z evropsko in svetovno književnostjo. Izdelanost standardne nemščine je obvladal Prešeren $v$ vseh izraznih tančinah. Intelektualne prvine in strokovno izražanje, tudi $v$ književnih teorijah, je bolje obvladal $v$ nemščini. Globoka lirična izpovedna moč pa je večja v slovenskih pesnitvah. V tem se tudi zaznava vloga jezika prve socializacije in drugega jezika. A tudi v slednjem je zaradi vseh življenjskih okoliščin Prešernovo izražanje dovolj močno. Na trenutke je enako slovenskemu, v nekaterih prvinah zaradi kompetence in duhovne podobe uporabnikov ter Prešerna samega semantično in slovniško strukturalno izjemno izdelano. Sama različnost prvin ob poglobljeni analizi ne daje povodov in pravic za pavšalne vrednostne sodbe, take, ki slone na individualnih vtisih. Razlike v recepciji pa obstajajo vedno. Poznavanje psiho-, socio- in etnolingvističnih vidikov (pri tem tudi geneze slovenskega jezika) omogoči boljše razpoznavanje razlik med obema jezikoma pri Prešernu in nam odpira tudi dodatne poglede na dejstva in fenomen velike umetniške moči Prešernovih slovenskih pesmi, a tudi dovoljšnje moči nemških pesmi. 